\title{
ENDEMIC BUBBLES GENERATED BY DELAYED BEHAVIORAL RESPONSE: GLOBAL STABILITY AND BIFURCATION SWITCHES IN AN SIS MODEL*
}

\author{
MAOXING LIU ${ }^{\dagger}$, EDUARDO LIZ ${ }^{\ddagger}$, AND GERGELY RÖST
}

\begin{abstract}
During infectious disease outbreaks, people may reduce their contact numbers or take other precautions to prevent transmission. The change in their behavior can be directly or indirectly triggered by the density of infected individuals in the population. In this paper, we investigate an SIS (susceptible-infected-susceptible) model where the transmission rate is a decreasing function of the prevalence of the disease (determined by a reduction function $h$ ), with the assumption that such a change in the transmission rate occurs with some time delay. We prove that if the basic reproduction number $R_{0}$ is less than one, then the disease-free equilibrium is globally asymptotically stable, while for $R_{0}>1$ a unique endemic equilibrium exists and the disease uniformly persists, regardless of the delay or the specific form of $h$. However, characterized by the shape of the response function $h$, various dynamics are possible if $R_{0}>1$. Roughly speaking, if $h$ is decreasing slowly (weak response), then the endemic equilibrium is absolutely globally asymptotically stable. When the slope of $h$ is larger (strong response), then the endemic equilibrium loses its stability and periodic orbits appear via Hopf bifurcation as $R_{0}$ increases. Further increasing $R_{0}$, the endemic equilibrium regains its stability, forming an interesting structure in the bifurcation diagram that we call an endemic bubble.
\end{abstract}

Key words. epidemic model, delay, global stability, stability switch, endemic bubble

AMS subject classifications. 34K20, 34D23, 92D30

DOI. $10.1137 / 140972652$

1. Introduction. Throughout history, epidemics have always influenced the behavior of people. In medieval times, they were fleeing from the plague. To see some more recent examples, during the outbreak of SARS in 2003 and influenza A (H1N1) in 2009, it was widely reported that the change in human behaviors was not only due to public measures, but also due to individual responses $[1,8]$. People can reduce the risk of infection by many ways: getting vaccination, canceling travel to dangerous regions, wearing face masks, using sanitizers, staying at home, etc. Recently, there has been a significant interest in modeling the aspects of health news, mass media, and psychological effects as applied to problems of epidemiology.

In traditional compartmental models, where the population is assumed to be large and homogeneous, the number of new infections per unit time is usually expressed by the standard incidence $\frac{\beta S(t) I(t)}{N(t)}$, where $\beta$ is the transmission rate and $S(t), I(t), N(t)$ stand for the number of individuals in the susceptible compartment, in the infected compartment, and in the population, respectively, at time $t$. A heuristic derivation

* Received by the editors June 12, 2014; accepted for publication (in revised form) October 31, 2014; published electronically January 13, 2015.

http://www.siam.org/journals/siap/75-1/97265.html

${ }^{\dagger}$ Department of Mathematics, North University of China, Taiyuan, Shanxi, People's Republic of China, 030051 (liumaoxing@126.com). This author's work was supported by European Research Council Starting Investigator grant 259559.

¥Departamento de Matemática Aplicada II, Universidade de Vigo, 36310 Vigo, Spain (eliz@dma. uvigo.es). This author's work was supported in part by the Spanish Government and FEDER, grant MTM2013-43404-P.

$\S$ Bolyai Institute, University of Szeged, H-6720, Szeged, Hungary (rost@math.u-szeged.hu). This author's work was supported by the European Union and the European Social Fund through project FuturICT.hu (grant TÁMOP 4.2.2.C-11/1/KONV-2012-0013) and Hungarian Scientific Research Fund OTKA K109782. 


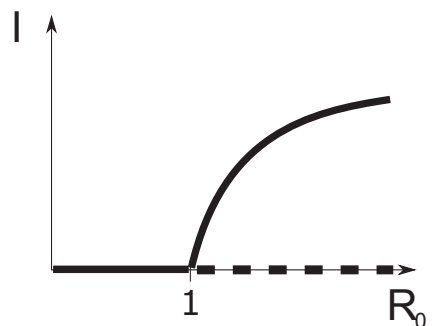

(a)

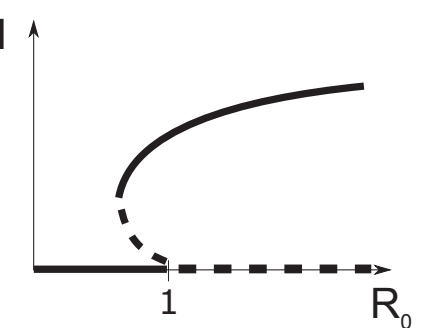

(b)

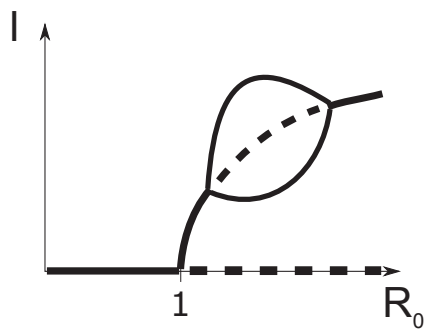

(c)

FiG. 1. Bifurcation diagrams: (a) forward bifurcation, (b) backward bifurcation, (c) endemic bubble.

of this term is the following: assume that an infected individual has $c$ contacts per unit time, each having the probability $p$ of transmitting the disease. Given that only contacts with susceptibles can result in a new infection, setting $\beta=p c$ and multiplying by the fraction $\frac{S(t)}{N(t)}$ of such contacts and the number of infected individuals $I(t)$, one obtains the standard incidence. Here it is assumed that the contact number $c$ and the transmission probability $p$ do not change. In reality, however, by the influence of media, public awareness, or personal experience, individuals apply precautionary measures that reduce the contact number or the transmission potential. The extent of such reactions naturally depends on the severity of the outbreak, i.e., the density of infection in the population. Accordingly, we can modify the standard incidence in a simple way by introducing a reduction factor $h(I)$; hence the incidence term becomes $\frac{\beta h(I) S(t) I(t)}{N(t)}$.

In recent decades, a large number of works (see, e.g., [2, 6, 14, 23, 24]) have been concerned with incorporating the effect of media or awareness into compartmental transmission models and investigating their impact on the disease dynamics. Various forms of $h(I)$ have been proposed in the literature, and the recent survey [4] identified three typical terms (which they call media functions), namely, $h(I)=\exp (-p I)$, $h(I)=1-I /(p+I)$, and $h(I)=1 /\left(1+p I^{2}\right)$, where the parameter $p$ represents the strength of the media effect. A more general class of nonlinear incidence (with $\left.h(I)=I^{a} /\left(1+b I^{c}\right)\right)$ was studied in $[10,11]$, and it was shown in [27] that modifying the standard incidence with $h(I)=1-p I$ is consistent with several real observations of infectious disease outbreaks. More recently even nonsmooth [25,26] and discontinuous $[12,13]$ cases have been studied, and the reporting delay was taken into account in [28].

In this work we consider an SIS (susceptible-infected-susceptible) setting with a general reduction term $h(I)$ that includes all the specific examples mentioned above and show that if this behavioral response is delayed, then this simple-looking system can exhibit surprising and interesting dynamics. One of our fundamental findings is the following. Considering the basic reproduction number $R_{0}$ as the key parameter, the bifurcation diagram typically looks like that of case (a) of Figure 1: for $R_{0}<$ 1 , only a disease-free equilibrium exists, which is stable, but its stability is lost at $R_{0}=1$, where a stable endemic equilibrium emerges via a transcritical bifurcation. For another class of models, so-called backward bifurcation occurs (see Figure 1 (b)), where two subcritical endemic equilibria coexist (a stable and an unstable), but for $R_{0}>1$ usually a unique endemic equilibrium exists which is stable. In this paper we show that for a class of SIS models with delayed behavioral adaptation, there is always a forward bifurcation at $R_{0}=1$. For $R_{0} \leq 1$ the disease-free equilibrium is 
always globally asymptotically stable, regardless of the particular choice of $h(y)$ and the value of the delay. For $R_{0}>1$, however, various dynamics are possible: as we increase the basic reproduction number, the endemic equilibrium can remain stable, or it can lose its stability at a critical point via Hopf bifurcation. In the latter case, we observe periodic oscillations only in an intermediate interval of reproduction numbers, and for large $R_{0}$ the endemic equilibrium always regains its stability. This interesting phenomenon results in a new bifurcation diagram for epidemiological models, which we call an endemic bubble, as depicted in Figure 1 (c). We illustrate this type of dynamical behavior with several examples.

2. The model and its basic properties. Our starting point is the standard susceptible-infected-susceptible (SIS) model without demography, which can be derived also as the mean-field approximation of homogeneous networks $[18,19]$, where infectious (I) individuals contaminate their susceptible (S) neighbors with some transmission rate, while the infected individuals recover and return to a susceptible state again. Then for the density of infected nodes one obtains the equation

$$
\frac{d I(s)}{d s}=-\mu I(s)+\beta\langle k\rangle I(s)(1-I(s)),
$$

where $\mu$ is the recovery rate of infected individuals. The second term represents the appearance of newly infected nodes. This is proportional to the density of infected nodes $(I(s))$, the transmission rate $\beta$, the number of links emanating from each node $\langle k\rangle$, and the probability that a given link points to a susceptible node, which is $1-I(s)$. Here $\mu, \beta$, and $\langle k\rangle$ are positive constants.

Next we introduce the behavioral response that reduces transmission by a smooth function $h(y)$, which satisfies

$$
h:[0,1] \rightarrow(0,1] \text { is } C^{1}, \quad h^{\prime}(y) \leq 0, \quad h(0)=1, \quad h(1)<1 .
$$

These hypotheses are reasonable: as $h$ represents a reduction factor in the transmission, it should take values not larger than one; the positivity requirement means it is not possible to completely eliminate transmission routes, while the monotone decreasing property expresses that the higher the density of infection, the more effort individuals make to minimize the risk of infection. The requirement $h(0)=1$ simply means that there is no reduction in the absence of the disease, and by $h(1)<1$ we exclude the trivial case when $h$ is constant one and there is no response whatsoever. Here we assume that such a response is activated with some delay $\sigma>0$, and the epidemic process in the present time $s$ is governed by a modified transmission rate that depends on the density of infection at time $s-\sigma$. This way we obtain the equation

$$
\frac{d I(s)}{d s}=-\mu I(s)+\beta\langle k\rangle h(I(s-\sigma)) I(s)(1-I(s)) .
$$

For the sake of simplicity, we rescale time by $y(t)=I\left(\mu^{-1} s\right)$; then, writing the equation for $y(t),(2.2)$ is transformed into

$$
\frac{d y(t)}{d t}=-y(t)+R_{0} h(y(t-\tau)) y(t)(1-y(t))
$$

where $\tau=\sigma \mu^{-1}$, and $R_{0}=\frac{\beta\langle k\rangle}{\mu}$ is called the basic reproduction number, which, as usual, expresses the expected number of secondary infections generated by a single infected individual introduced into a wholly susceptible population. In what follows 
we study (2.3) and refer to $y(t)$ as the density of infection at time $t$. Let $C$ be the Banach space of continuous real functions on $[-\tau, 0]$, equipped with the supremum norm. Considering the biological interpretation of the model, the feasible phase space is the subset of $C$ that consists of functions with the range in the interval $[0,1]$; let us denote this set by $X$.

Proposition 2.1. For any initial function $\phi \in C$ with $\phi(s) \in[0,1]$ for all $s \in[-\tau, 0],(2.3)$ has a unique global solution $y^{\phi}(t)$ with $y(t) \in[0,1]$ for all $t>0$. If $R_{0} \leq 1$, the zero solution is globally asymptotically stable, i.e., $y^{\phi}(t) \rightarrow 0$ as $t \rightarrow \infty$. If $R_{0}>1$, the zero solution is unstable and (2.3) has a unique endemic equilibrium $\bar{y}$, with $\bar{y}<1-1 / R_{0}$.

Proof. The existence and uniqueness follows from the smoothness assumption on $h$. The invariance of $X$ is a consequence of $y^{\prime}(t)=0$ for $y(t)=0$ and $y^{\prime}(t)=-1<0$ for $y(t)=1$. The boundedness implies the global existence as well.

To obtain the equilibria of $(2.3)$, set $y(t)=y(t-\tau)=\bar{y}$, and let the right-hand side of (2.3) be zero, so

$$
R_{0} h(\bar{y}) \bar{y}(1-\bar{y})=\bar{y} .
$$

Clearly $\bar{y}=0$ is always an equilibrium. Let $H(y):=R_{0} h(y)(1-y)$. Then $\bar{y}>0$ is a positive equilibrium if and only if $H(\bar{y})=1$. Given that $H(y)$ is monotone decreasing, $H(0)=R_{0}$, and $H(1)=0$, a positive equilibrium exists if and only if $R_{0}>1$, and it is unique when it exists. From the observation

$$
H\left(1-\frac{1}{R_{0}}\right)=R_{0} h\left(1-\frac{1}{R_{0}}\right) \frac{1}{R_{0}} \leq 1,
$$

we conclude that $\bar{y} \leq 1-1 / R_{0}$.

From the comparison equation

$$
y^{\prime}(t) \leq-y(t)+R_{0} y(t)(1-y(t))=\left(R_{0}-1\right) y(t)-R_{0} y(t)^{2},
$$

we can easily get that the zero equilibrium is globally asymptotically stable if $R_{0} \leq 1$. Furthermore, the linear variational equation of (2.3) at the zero solution is

$$
\frac{d y(t)}{d t}=\left(R_{0}-1\right) y(t)
$$

Thus, if $R_{0}>1$, the zero solution is unstable.

Clearly if $\phi \in X$ with $\phi(0)=0$, then $y^{\phi}(t)=0$ for all $t>0$. We say that a solution is nontrivial if $\phi(0)>0$.

3. Permanence. We say that system (2.3) is permanent if nontrivial solutions are uniformly bounded away from the boundary of the feasible phase space; i.e., there are constants $m>0$ and $M<1$ such that for any $\phi \in X$ with $\phi(0)>0$, there is a $T=T(\phi)$ such that $m \leq y^{\phi}(t) \leq M$ for all $t>T$. We introduce the notation

$$
y^{\infty}:=\limsup _{t \rightarrow \infty} y(t), \quad y_{\infty}:=\liminf _{t \rightarrow \infty} y(t), \quad y_{*}:=\bar{y} e^{-\tau}, \quad y^{*}:=1-\frac{1}{R_{0}} .
$$

THEOREM 3.1. If $R_{0}>1$ and $h(y)$ satisfies $(H)$, then system (2.1) is permanent. In particular, for every nontrivial solution $y(t)$, the relation

$$
y_{*} \leq y_{\infty} \leq \bar{y} \leq y^{\infty} \leq y^{*}
$$

holds. 
Proof. Consider an arbitrary nontrivial solution $y(t)$. The proof is divided into four steps.

(i) $y^{\infty} \leq y^{*}$. According to the fluctuation lemma (Lemma A.5 in [22]), there exists a sequence $\left(t_{n}\right)$ such that $t_{n} \rightarrow \infty$ as $n \rightarrow \infty$, and $y^{\prime}\left(t_{n}\right) \rightarrow 0, y\left(t_{n}\right) \rightarrow y^{\infty}$ as $n \rightarrow \infty$. Passing to the limit in

$$
y^{\prime}\left(t_{n}\right) \leq-y\left(t_{n}\right)+R_{0} y\left(t_{n}\right)\left(1-y\left(t_{n}\right)\right),
$$

where we used $h\left(y\left(t_{n}\right)-\tau\right) \leq 1$, we obtain

$$
0 \leq-y^{\infty}+R_{0} y^{\infty}\left(1-y^{\infty}\right)
$$

and thus

$$
y^{\infty} \leq 1-\frac{1}{R_{0}}=y^{*}<1 .
$$

(ii) $y^{\infty} \geq \bar{y}$. Suppose the contrary, i.e., $y^{\infty}<\bar{y}$; then for any nontrivial solution $y(t)$ there is a $T>0$ such that $y(t)<\bar{y}$ for all $t>T$. Then for all $t>T+\tau$, we have $h(y(t-\tau)) \geq h(\bar{y})$ and $1-y(t) \geq 1-\bar{y}$, and thus

$$
y^{\prime}(t) \geq y(t)\left[-1+R_{0} h(\bar{y})(1-\bar{y})\right]=0 .
$$

Then $y(t)$ is monotone increasing and thus converging; therefore necessarily $y(t) \rightarrow \bar{y}$ as $t \rightarrow \infty$, which means $y^{\infty}=\bar{y}$, a contradiction. Hence, $y^{\infty} \geq \bar{y}$.

(iii) $y_{\infty} \leq \bar{y}$. We proceed similarly as in step (ii): if $y(t)>\bar{y}$ for all $t>T$ with some $T$, then the relation

$$
y^{\prime}(t) \leq y(t)\left[-1+R_{0} h(\bar{y})(1-\bar{y})\right]=0
$$

holds for all $t>T+\tau$, and thus $y_{\infty} \leq \bar{y}$.

(iv) $y_{\infty} \geq y_{*}$. We claim that for any $\varepsilon>0, y_{\infty} \geq e^{-\tau}(\bar{y}-\varepsilon)$ holds. Since $y^{\infty} \geq \bar{y}$, there exists a sequence $\left(s_{k}\right)$ such that $s_{k} \rightarrow \infty$ and $y\left(s_{k}\right)>\bar{y}-\varepsilon$. Consider any $t_{*}>s_{1}$ with $y(t)<\bar{y}-\varepsilon$. If there is no such $t_{*}$, then obviously $y_{\infty} \geq \bar{y}-\varepsilon>e^{-\tau}(\bar{y}-\varepsilon)$. Otherwise there is a $k$ such that $s_{k}<t_{*}<s_{k+1}$ and there is a maximal interval $J=(\alpha, \beta) \subset\left[s_{k}, s_{k+1}\right]$ such that $t_{*} \in J$ and, for any $t \in J, y(t)<\bar{y}-\varepsilon$. By continuity of solutions, $y(\alpha)=y(\beta)=\bar{y}-\varepsilon$. We show that for any $t \in J$, the inequality $y(t) \geq e^{-\tau}(\bar{y}-\varepsilon)$ holds. Indeed, from the estimate $y^{\prime}(t)>-y(t)$ one has $y(t)>e^{-(t-\alpha)} y(\alpha)$, which gives $y(t) \geq e^{-\tau}(\bar{y}-\varepsilon)$ whenever $t \in[\alpha, \alpha+\tau]$. If $\beta>\alpha+\tau$, then for $t>\alpha+\tau$ we have $y^{\prime}(t) \geq 0$, and thus $y(t) \geq y(\alpha+\tau) \geq e^{-\tau}(\bar{y}-\varepsilon)$. Letting $\varepsilon \rightarrow 0$, we finish the proof.

\section{Global stability and Hopf bifurcation.}

4.1. Local stability of the endemic equilibrium. In the following, we consider the stability of $\bar{y}$. Let $G(u, v)=-u+R_{0} h(v) u(1-u), x=y-\bar{y}$, and linearize system (2.3) at the equilibrium $\bar{y}$ to obtain

$$
\frac{d x(t)}{d t}=A x(t)+B x(t-\tau)
$$

where

$$
A=G_{u}(\bar{y}, \bar{y}), \quad B=G_{v}(\bar{y}, \bar{y}) .
$$

Copyright (c) by SIAM. Unauthorized reproduction of this article is prohibited. 
A straightforward calculation, using $R_{0} h(\bar{y})(1-\bar{y})=1$, gives

$$
A=-1+R_{0} h(\bar{y})(1-2 \bar{y})=\frac{-\bar{y}}{1-\bar{y}}<0, \quad B=R_{0} \bar{y}(1-\bar{y}) h^{\prime}(\bar{y})<0 .
$$

According to the well-known stability result of scalar delay differential equations with a single delay (see, for example, Theorem 4.7 in [22]), we immediately find the following theorem.

TheOREM 4.1. (a) If

$$
h^{\prime}(\bar{y}) \geq-R_{0} h^{2}(\bar{y})
$$

then the positive equilibrium $\bar{y}$ is asymptotically stable.

(b) If $h^{\prime}(\bar{y})<-R_{0} h^{2}(\bar{y})$, there exists a $\tau^{*}>0$, such that the positive equilibrium $\bar{y}$ is asymptotically stable for $0<\tau<\tau^{*}$ and unstable for $\tau>\tau^{*}$, where

$$
\tau^{*}=\frac{1}{\omega^{*}} \arccos \left(-\frac{A}{B}\right), \quad \omega^{*}=\sqrt{B^{2}-A^{2}} .
$$

The next observation shows that the endemic equilibrium is asymptotically stable for both small (in the sense that it is close to 1) and large basic reproduction numbers.

Proposition 4.2. (a) For any $h(y)$ satisfying $(H)$, there exists an $R^{*}$, such that if $R_{0}>R^{*}$, then the positive equilibrium is asymptotically stable for all values of the delay.

(b) For any $h(y)$ satisfying $(H)$ and $\left|h^{\prime}(0)\right|<1$, there is an $R_{*}$, such that if $1<R_{0}<R_{*}$, then the positive equilibrium is asymptotically stable for all values of the delay.

(c) For any fixed $\tau$ and $h(y)$ satisfying $(H)$ and $\left|h^{\prime}(0)\right| \geq 1$, there is an $R_{*}=$ $R_{*}(\tau, h)$, such that if $1<R_{0}<R_{*}$, then the positive equilibrium is asymptotically stable.

Proof. (a) As $h(y)$ is $C^{1}$, we can choose $R^{*}=\max _{y \in[0,1]} \frac{\left|h^{\prime}(y)\right|}{h(y)^{2}}$; then for $R_{0}>R^{*}$, the condition (4.2) holds.

(b) It follows from $\bar{y} \leq 1-1 / R_{0}$ that $\lim _{R_{0} \rightarrow 1^{+}} \bar{y}=0$ and $\lim _{R_{0} \rightarrow 1^{+}} h(\bar{y})=1$; thus (4.2) holds if $R_{0}$ is sufficiently close to 1, independently of the delay.

(c) It is sufficient to show that the critical delay, defined in (4.3), is tending to infinity as $R_{0}$ is approaching 1 from the right. From $A<0$ and $B<0$ it follows that $\tau^{*} \in\left(\frac{\pi}{2 \omega^{*}}, \frac{\pi}{\omega^{*}}\right)$. As $R_{0} \rightarrow 1^{+}$, we have $A \rightarrow 0, B \rightarrow 0$; thus $\omega^{*} \rightarrow 0$ and indeed $\tau^{*} \rightarrow \infty$.

4.2. Global stability of the endemic equilibrium. We note that in case (b) of Theorem 4.1, system (2.1) goes through a Hopf-bifurcation at $\tau=\tau^{*}$. From our simulations it seems that this bifurcation is always supercritical and a stable limit cycle appears. There are well-established methods to determine the direction of the bifurcation for this kind of equation, but we are not concerned with such calculations here. More importantly, we get some global stability conditions for the positive equilibrium $\bar{y}$ of (2.3) using the approach of [7] and [17]. For the four choices we make for the map $h$, we will show that the sharp delay-independent condition for local stability given in (4.2) is also sufficient to ensure the global stability of $\bar{y}$ (sometimes with the strict inequality).

In [7], the following delay differential equation was considered:

$$
y^{\prime}(t)=f_{1}(y(t-\tau)) g_{2}(y(t))-f_{2}(y(t-\tau)) g_{1}(y(t))
$$

Copyright $@$ by SIAM. Unauthorized reproduction of this article is prohibited. 
where $f_{i}, g_{i}$ are continuous nonnegative functions, $f_{i}(y)>0, g_{i}(y)>0$ for $y>0$, $i=1,2$, and $g(y)=g_{1}(y) / g_{2}(y)$ is strictly increasing. It is also assumed that there is a unique positive equilibrium $\bar{y}$ of (4.4), which is the only solution of equation $f(y)=g(y)$, where $f(y)=f_{1}(y) / f_{2}(y)$. Moreover, $f(y)>g(y)$ for $y \in(0, \bar{y})$, and $f(y)<g(y)$ for $y>\bar{y}$.

Consider the map $F(y)=g^{-1}(f(y))$. It is clear that $\bar{y}$ is a fixed point of $F$. Theorem 3 in [7] ensures that if $\bar{y}$ is an attracting point of $F$ with immediate domain of attraction $J_{0}$ and $\phi:[-\tau, 0] \rightarrow J_{0}$ is continuous, then the solution $y=y(\cdot, \phi)$ of (4.4) with $y(t)=\phi(t)$ for all $t \in[-\tau, 0]$ satisfies $\lim _{t \rightarrow \infty} y(t, \phi)=\bar{y}$.

It is clear that (2.3) belongs to this class of delay differential equations, with $f_{1}(y)=h(y), f_{2}(y)=1, g_{1}(y)=y$, and $g_{2}(y)=R_{0} y(1-y)$. Thus, $f(y)=h(y)$, $g(y)=1 /\left(R_{0}(1-y)\right)$, and

$$
F(y)=g^{-1}(f(y))=1-\frac{1}{R_{0} h(y)} .
$$

In view of Theorem 2.2, we can restrict our analysis to initial functions $\phi:[-\tau, 0]$ $\rightarrow\left[0, y^{*}\right]$, where $y^{*}=1-1 / R_{0}$. Since $F$ is strictly decreasing and $F(0)=y^{*}$, it follows that $F$ maps $\left[0, y^{*}\right]$ in $\left[0, y^{*}\right]$ if $F\left(y^{*}\right) \geq 0$, that is, if the following condition holds:

$$
h\left(1-\frac{1}{R_{0}}\right) \geq \frac{1}{R_{0}} \text {. }
$$

The next theorem follows from Theorem 3 in [7] (see also $[5,15,20]$ ). As usual, we denote by $F^{k}$ the corresponding power of $F$ under composition, that is,

$$
F^{k}=\underbrace{F \circ \cdots \circ F}_{k} .
$$

Theorem 4.3. Assume that conditions $(H)$ and (4.6) hold. If $\lim _{k \rightarrow \infty} F^{k}(y)=\bar{y}$ for all $y \in\left[0, y^{*}\right]$, then all solutions of $(2.3)$ converge to $\bar{y}$.

Next we give two corollaries of Theorem 4.3 that will be very useful in the applications. We recall that the Schwarzian derivative of a $C^{3}$ map $F$ is defined by

$$
(S F)(x)=\frac{F^{\prime \prime \prime}(x)}{F^{\prime}(x)}-\frac{3}{2}\left(\frac{F^{\prime \prime}(x)}{F^{\prime}(x)}\right)^{2} \text {. }
$$

The following well-known result follows from Singer's paper [21]; see, e.g., Theorem 1 in [16].

Proposition 4.4. Assume that $F: J \rightarrow J$ is a $C^{3}$ map defined in a bounded real interval $J$, such that $F^{\prime}(y)<0$ and $(S F)(y)<0$ for all $y \in J$. Let $\bar{y}$ be the only fixed point of $F$. If $F^{\prime}(\bar{y}) \geq-1$, then $\lim _{k \rightarrow \infty} F^{k}(y)=\bar{y}$ for all $y \in J$.

Corollary 4.5. Assume that conditions $(H),(4.6)$, and (4.2) hold. If $(S h)(x)<$ 0 for all $x \in\left(0, y^{*}\right)$, then the positive equilibrium $\bar{y}$ of (2.3) is globally asymptotically stable.

Proof. Condition (4.2) implies that $\bar{y}$ is locally asymptotically stable for (2.3). Moreover, it is easy to verify that $F^{\prime}(\bar{y}) \geq-1$ if and only if (4.2) holds. Since $F(y)=G(h(y))$, where $G(y)=g^{-1}(y)=1-1 /\left(R_{0} y\right)$, it is easy to check that $(S F)(y)=(S h)(y)$ (see, e.g., [21, p. 262]). Next, since $F$ is strictly decreasing, the result follows from Proposition 4.4 and Theorem 4.3.

COROLlary 4.6. Assume that conditions $(H)$, (4.6), and (4.2) hold, with the strict inequality in the latter. If $h$ is a real Möbius transformation, then the positive equilibrium $\bar{y}$ of (2.3) is globally asymptotically stable.

Copyright (c) by SIAM. Unauthorized reproduction of this article is prohibited. 
Proof. In this case $F(y)=G(h(y))$ is a real Möbius transformation. Thus, condition $F^{\prime}(\bar{y})>-1$ implies that $\bar{y}$ is a global attractor of $F$; see, for example, Corollary 7 in [3].

4.3. Application 1: $\boldsymbol{h}(\boldsymbol{y})=\mathbf{1}-\boldsymbol{p} \boldsymbol{y}$. In this case, (2.3) writes

$$
y^{\prime}(t)=-y(t)+R_{0} y(t)(1-y(t))(1-p y(t-\tau)) .
$$

Such a transmission term (without delay) was used in [27]. Notice that $(H)$ holds if and only if $0<p<1$. If $R_{0}>1$, then (4.7) has a unique positive equilibrium in $(0,1)$ defined by

$$
\bar{y}=\frac{p+1-\sqrt{(p-1)^{2}+4 p / R_{0}}}{2 p} .
$$

Next, it is easy to prove that (4.2) and (4.6) hold for all $p<1$, with strict inequality in (4.2). Thus, an application of Corollary 4.6 provides the following result.

THEOREM 4.7. Assume that $0<p<1$. If $R_{0}>1$, then the positive equilibrium $\bar{y}$ of (4.7) is globally asymptotically stable.

Theorem 4.7 ensures that the endemic equilibrium $\bar{y}$ is globally stable if $R_{0}>1$, regardless of the value of $p$. However, the value of $p$ influences the size of $\bar{y}$; see Figure 2.

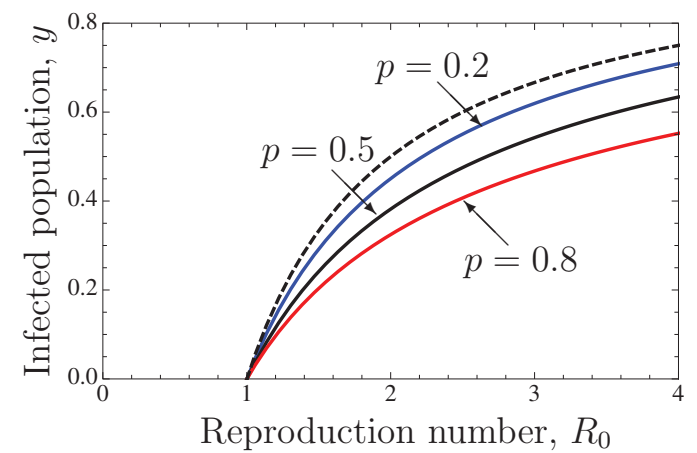

FIG. 2. Endemic equilibrium $\bar{y}$ of (4.7), as $R_{0}$ increases, for different values of $p$. The dashed curve corresponds to the upper bound $1-1 / R_{0}$ for $\bar{y}$ (see Proposition 2.1).

5. Endemic bubbles. In contrast with the previous example, in most of the applications the endemic equilibrium is unstable for some values of the significant parameters $p, \tau$, and $R_{0}$. In this section, we report three cases that exhibit endemic bubbles.

5.1. Application 2: $\boldsymbol{h}(\boldsymbol{y})=\frac{\mathbf{1}}{\mathbf{1 + p \boldsymbol { y }}}$. Note that with $\tilde{p}=1 / p$, we can write

$$
h(y)=\frac{1}{1+p y}=\frac{\tilde{p}}{\tilde{p}+y}=1-\frac{y}{\tilde{p}+y},
$$

which is exactly the third identified media function in [4]. However, to keep consistent the meaning of parameter $p$ as the intensity of the behavioral response, we use the form $h(y)=1 /(1+p y)$. In this case, (2.3) can be written as

$$
y^{\prime}(t)=-y(t)+\frac{R_{0} y(t)(1-y(t))}{1+p y(t-\tau)} .
$$

Copyright (C) by SIAM. Unauthorized reproduction of this article is prohibited. 
Condition $(H)$ is satisfied if and only if $p>0$. If $R_{0}>1$, then (5.1) has a unique positive equilibrium in $(0,1)$ defined by

$$
\bar{y}=\frac{R_{0}-1}{R_{0}+p} .
$$

Next, it is easy to prove that (4.2) and (4.6) hold if and only if $p \leq R_{0}$, and if $p<R_{0}$, then the inequality is strict in (4.2). Thus, an application of Theorem 4.1 and Corollary 4.6 provides the following result.

TheOREM 5.1. Assume that $p>0$ and $R_{0}>1$.

(1) If $0<p<R_{0}$, then the positive equilibrium $\bar{y}$ of (5.1) is globally asymptotically stable.

(2) If $p>R_{0}$, then there exists $\tau^{*}>0$, such that the positive equilibrium $\bar{y}$ is asymptotically stable for $0<\tau<\tau^{*}$ and unstable for $\tau>\tau^{*}$, where

$$
\tau^{*}=\frac{R_{0}(p+1)}{\left(R_{0}-1\right) \sqrt{p^{2}-R_{0}^{2}}} \arccos \left(\frac{-R_{0}}{p}\right) .
$$

We notice that Theorem 5.1 provides explicit stability conditions in terms of the involved parameters. In particular, it is easy to check, taking the limit as $p \rightarrow \infty$ in (5.2), that for $R_{0}>1$ fixed, the endemic equilibrium $\bar{y}$ is asymptotically stable for all values of $p$ if $\tau$ is small enough, specifically, if $\tau<\pi R_{0} /\left(2\left(R_{0}-1\right)\right)$.

Next, we use Theorem 5.1 to show the stability regions for (5.1) as a function of the three parameters. We first observe that both increasing $\tau$ and increasing $p$ destabilize the endemic equilibrium $\bar{y}$. Figure 3 shows this fact for $R_{0}=2$.

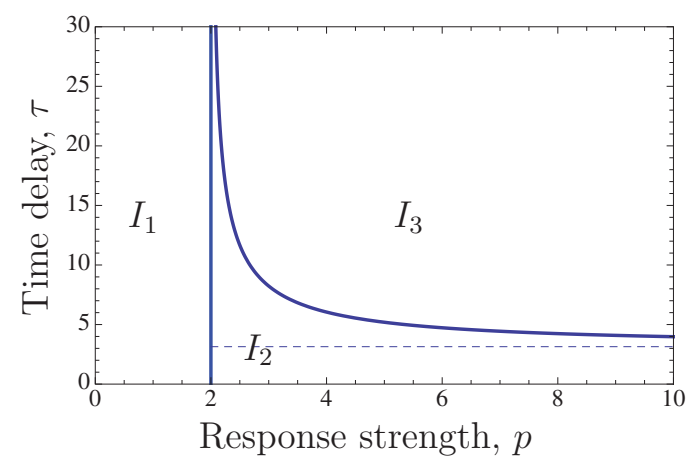

FIG. 3. Two-parameter stability diagram of (5.1) for fixed $R_{0}=2$, showing the dependence on the response strength $p$ and time delay $\tau$ : absolute global stability of $\bar{y}$ in region $I_{1}(p<2)$; local asymptotic stability of $\bar{y}$ in $I_{2}$; and instability of $\bar{y}$ in $I_{3}$. The dashed line $\tau=\pi$ emphasizes that small time delays are not able to destabilize $\bar{y}$.

The monotone character of the stability boundary shown in Figure 3 is lost when we choose the reproduction number $R_{0}$ as a bifurcation parameter. This fact is easily observable in Figure 4: the endemic equilibrium $\bar{y}$ is stable for small enough and large enough values of $R_{0}$, but it loses its stability for intermediate values of $R_{0}$.

Next we take the basic reproduction number $R_{0}$ as the key parameter to show its incidence in the dynamics. For this, we fix $p=4$ and $\tau=10$ in (5.1). For an initial condition $\phi(t)=0.2, t \in[-\tau, 0]$, we plot the minimum and the maximum values of the solution $x(t, \phi)$ between $t=1400$ and $t=1500$, once the transients have died out. 

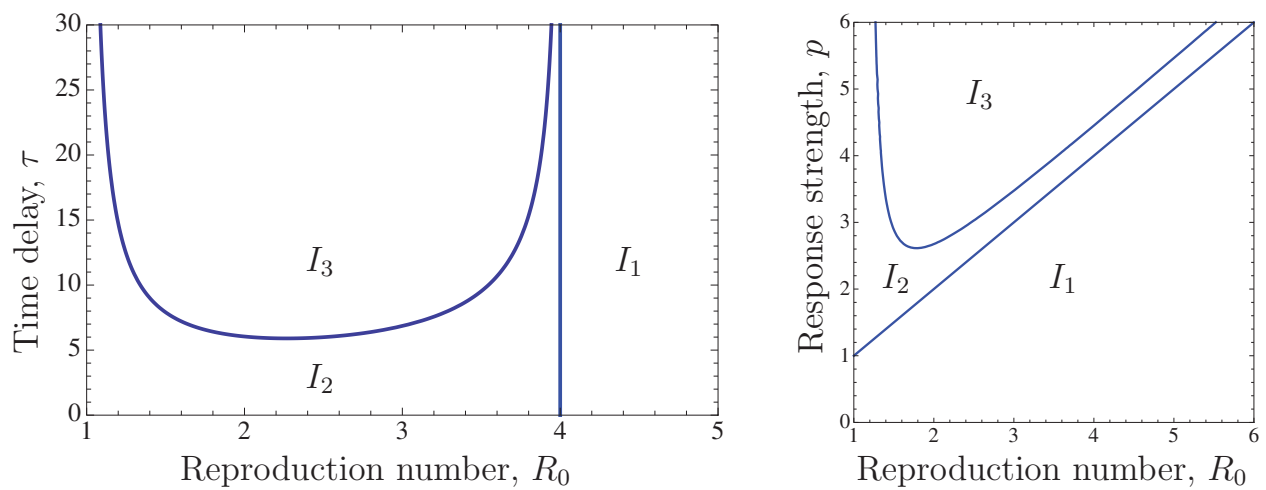

FIG. 4. Two-parameter stability diagrams for (5.1). Left: for fixed $p=4$, dependence on the reproduction number $R_{0}$ and time delay $\tau$. Right: for fixed $\tau=10$, dependence on the reproduction number $R_{0}$ and the response strength $p$. In both cases, region $I_{1}\left(p<R_{0}\right)$ corresponds to the absolute global stability of $\bar{y} ; \bar{y}$ is locally asymptotically stable in $I_{2}$; and $\bar{y}$ is unstable in $I_{3}$.

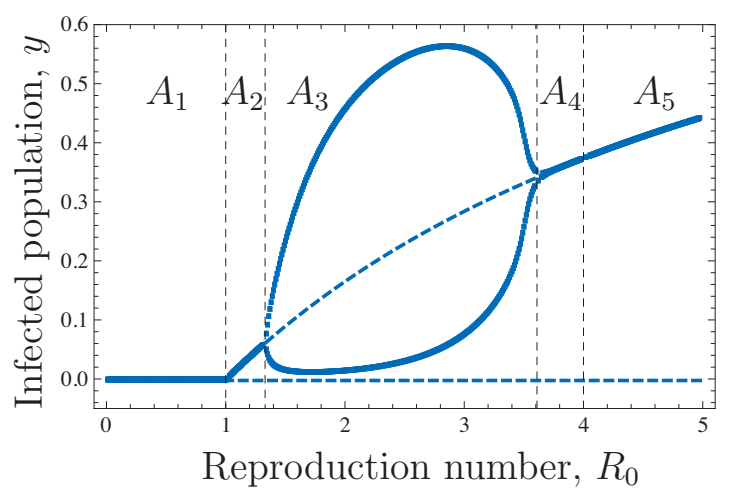

FiG. 5. Bifurcation diagram for (5.1) displaying an endemic bubble. The different regions correspond to different dynamics: $A_{1}$ for global stability of the disease-free equilibrium $0 ; A_{2}$ and $A_{4}$ for local stability of the endemic equilibrium $\bar{y} ; A_{3}$ for sustained oscillations; and $A_{5}$ for global stability of $\bar{y}$. Note that for regions $A_{2}$ and $A_{4}$ the simulations suggest the global attractivity of the endemic equilibrium for positive solutions; however, our analysis proves only local stability. For this reason we separated $A_{4}$ from $A_{5}$, where global asymptotic stability is rigorously proven. Thick dashed lines correspond to the equilibria 0 and $\bar{y}$ when they are unstable.

In this way, we get the bifurcation diagram in Figure 5, which we call an endemic bubble (compare with Figure 1).

To illustrate how the long-term behavior of the solutions of (5.1) changes as $R_{0}$ is increased, we plot in Figure 6 the solution $y(t, \phi)$ for values of $R_{0}$ in the different regions of Figure 5.

5.2. Application 3: $\boldsymbol{h}(\boldsymbol{y})=e^{-p y}$. This is the case of the first identified media function in [4], and now (2.3) can be written as

$$
y^{\prime}(t)=-y(t)+R_{0} y(t)(1-y(t)) e^{-p y(t-\tau)} .
$$

It is clear that condition $(H)$ holds for all $p>0$. Moreover, $(S h)(x)=-p^{2} / 2<0$ for all $x \in \mathbb{R}$. 

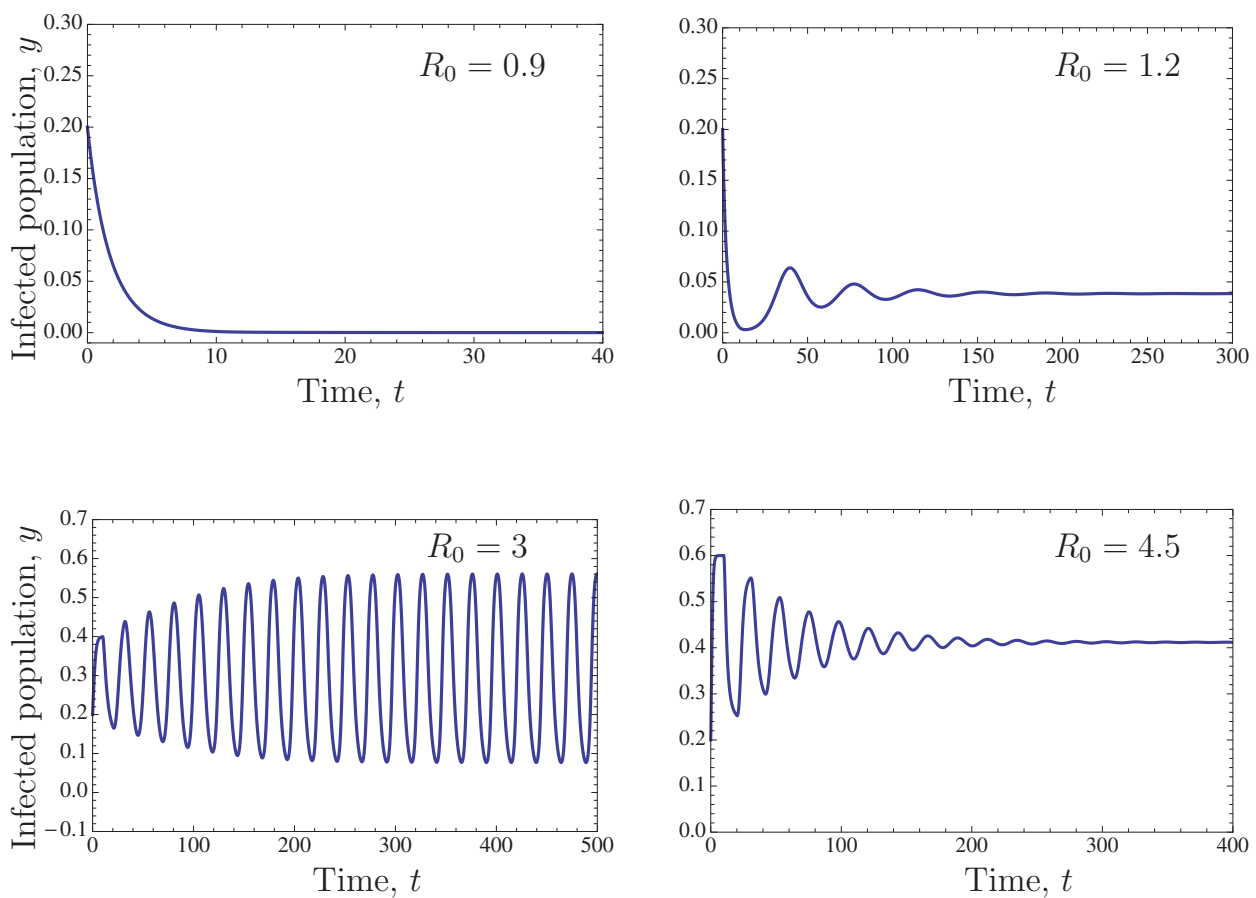

FIG. 6. Solutions of (5.1) with $p=4, \tau=10$, and different values of $R_{0}$, corresponding to regions $A_{1}\left(R_{0}=0.9\right), A_{2}\left(R_{0}=1.2\right), A_{3}\left(R_{0}=3\right)$, and $A_{5}\left(R_{0}=4.5\right)$ in Figure 5 . For $R_{0}<1$ all solutions converge to 0 . For $R_{0}>1$, the endemic equilibrium $\bar{y}$ is first stable, then loses its asymptotic stability in a Hopf bifurcation at $R_{0} \approx 1.34$, and regains it in a new Hopf bifurcation at $R_{0} \approx 3.55$. For intermediate values of $R_{0}$, sustained oscillations are observed from numerical simulations.

Before stating a global stability result for (5.3), we need some auxiliary lemmas. LEMMA 5.2.

(1) The local stability condition (4.2) holds for (5.3) if and only if

$$
R_{0} \geq p e^{p-1} .
$$

(2) Condition (4.6) holds for (5.3) if and only if

$$
R_{0} \geq e^{p\left(1-1 / R_{0}\right)} .
$$

Proof. The positive equilibrium $\bar{y}$ of (5.3) is the only zero of the map $S(x)=$ $e^{p x}-R_{0}(1-x)$. Direct calculations show that the stability condition (4.2) is equivalent to the inequality $\bar{y} \geq 1-1 / p$. Since $S(0)=1-R_{0}<0$ and $S(1)=e^{p}>0$, it follows that the inequality $\bar{y} \geq 1-1 / p$ can be written as $S(1-1 / p) \leq 0$, that is, $R_{0} \geq p e^{p-1}$.

The proof of the second statement of the lemma follows immediately from the inequality $h\left(1-1 / R_{0}\right) \geq 1 / R_{0}$.

Our next result relates conditions (5.4) and (5.5).

LEMMA 5.3.

(1) If $p \leq 1$, then both conditions (5.4) and (5.5) hold.

(2) If $p>1$, then condition (5.4) implies (5.5).

Proof. The first claim of the lemma follows from the elementary inequalities $p e^{p-1} \leq 1$ for all $p \leq 1$, and $R_{0} e^{1 / R_{0}-1} \geq 1$ for all $R_{0}>0$. 
Now assume $p>1$ and $R_{0} \geq p e^{p-1}$. Then $R_{0} \geq p$ also holds, and we need to show $s\left(R_{0}\right) \geq s(1)$, where $s(x)=x e^{\frac{p}{x}}$. Notice that the function $s(x)$ is increasing for $x \geq p$, since $s^{\prime}(x)=e^{\frac{p}{x}}\left(1-\frac{p}{x}\right)$. Therefore, $s\left(R_{0}\right) \geq s\left(p e^{p-1}\right)=p e^{p-1} e^{e^{1-p}}$. Let $w(p)=p \exp \left(e^{1-p}-1\right)$; then $w(1)=1$ and $w^{\prime}(p)=\exp \left(e^{1-p}-1\right) e^{1-p}\left(e^{p-1}-p\right) \geq 0$, and thus $w(p) \geq 1$ for all $p \geq 1$. Since $s\left(p e^{p-1}\right)=e^{p} w(p) \geq e^{p}=s(1)$, the proof is finished.

The following stability result for (5.3) is a direct consequence of Corollary 4.5 and Lemmas 5.2 and 5.3.

TheOREM 5.4. Assume that $p>0$. If $R_{0}>1$, then (5.3) has a unique positive equilibrium $\bar{y} \in(0,1)$, which is the only solution of equation $e^{p x}=R_{0}(1-x)$.

(1) If condition (5.4) holds, then the positive equilibrium $\bar{y}$ is globally asymptotically stable.

(2) If condition (5.4) does not hold, then there exists $\tau^{*}>0$ such that $\bar{y}$ is asymptotically stable for $0<\tau<\tau^{*}$ and unstable for $\tau>\tau^{*}$, where $\tau^{*}$ is given by (4.3).

Using Theorem 5.4, it is easy to produce stability diagrams as we did for our previous application. Since they are qualitatively similar, we do not include them.

5.3. Application 4: $\boldsymbol{h}(\boldsymbol{y})=\frac{1}{1+(\boldsymbol{p y})^{n}}$. The special case $n=2$ is the second identified media function in [4]; more generally, here we write (2.3) as

$$
y^{\prime}(t)=-y(t)+\frac{R_{0} y(t)(1-y(t))}{1+(p y(t-\tau))^{n}} .
$$

Condition $(H)$ holds if and only if $p>0$. We will also assume that $n>1$, which in particular implies that $(S h)(y)=\frac{1-n^{2}}{2 y^{2}}<0$ for all $y>0$. Notice that the case $n=1$ gives our second application (5.1), for which the Schwarzian derivative is not negative.

To illustrate the influence of $p$ in the shape of function $h$, we plot the graph of $h$ for $n=2$ and various values of $p$ in Figure 7 .

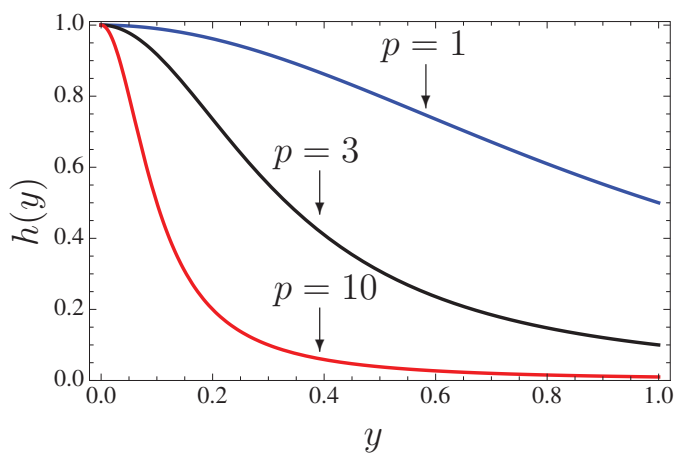

FIG. 7. Influence of parameter $p$ on the shape of function $h(y)$ in (5.6).

As in the previous case, to obtain stability criteria for (5.6), we need some preliminary lemmas.

LEMMA 5.5. The local stability condition (4.2) holds for (5.6) if and only if

$$
\left(R_{0}-1\right) R_{0}^{\frac{n}{1-n}} p^{\frac{n}{n-1}} \leq \frac{1}{n^{\frac{n}{n-1}}}+\frac{1}{n^{\frac{1}{n-1}}} .
$$

Copyright (C) by SIAM. Unauthorized reproduction of this article is prohibited. 
Proof. The positive equilibrium $\bar{y}$ of (5.6) is the only solution of equation $(p x)^{n}+$ $1=R_{0}(1-x)$. Direct calculations show that (4.2) holds if and only if $\bar{y}$ satisfies the inequality

$$
\bar{y} \leq\left(\frac{R_{0}}{n p^{n}}\right)^{\frac{1}{n-1}} .
$$

Define the map $S(x)=(p x)^{n}+R_{0} x+1-R_{0}$ in such a way that $\bar{y}$ is the only solution of the equation $S(x)=0$. Since $S(0)=1-R_{0}<0$, and $S(1)=p^{n}+1>0$, (5.8) holds if and only if

$$
S\left(\left(R_{0} /\left(n p^{n}\right)\right)^{1 /(n-1)}\right) \geq 0 .
$$

It is easy to check that the last inequality is equivalent to (5.7).

The proof of the following result is straightforward.

Lemma 5.6. Condition (4.6) holds for (5.6) if and only if

$$
\left(R_{0}-1\right) R_{0}^{\frac{n}{1-n}} p^{\frac{n}{n-1}} \leq 1 .
$$

Next, the following lemma ensures that (4.6) holds for (5.6) whenever the positive equilibrium is locally asymptotically stable.

LEMma 5.7. The following inequality holds for all $n>1$ :

$$
\frac{1}{n^{\frac{n}{n-1}}}+\frac{1}{n^{\frac{1}{n-1}}} \leq 1
$$

Proof. We start with the Bernoulli inequality $(1+x)^{r} \geq 1+r x$, which holds for every $r \geq 1$ and $x>-1$. Let $z>0$ and choose $r=1+1 / z$ and $x=z$; then we have $(1+z)^{(1+1 / z)} \geq 2+z$, or alternatively $(1+z)^{1 / z} \geq 1+1 /(1+z)$. The latter is equivalent to

$$
1 \geq(1+z)^{-\frac{1}{z}}\left(1+\frac{1}{1+z}\right)=\frac{1}{(1+z)^{\frac{1}{z}}}+\frac{1}{(1+z)^{\frac{1}{z}+1}} .
$$

Now substitute $n=z+1$ to find

$$
1 \geq \frac{1}{n^{\frac{1}{n-1}}}+\frac{1}{n^{\frac{1}{n-1}+1}}=\frac{1}{n^{\frac{1}{n-1}}}+\frac{1}{n^{\frac{n}{n-1}}}
$$

We are now in a position to state a stability result for the positive equilibrium of (5.6). Its proof is a direct consequence of Corollary 4.5 and Lemmas 5.5, 5.6, and 5.7.

TheOrem 5.8. Assume that $p>0$ and $n>1$. If $R_{0}>1$, then (5.6) has a unique positive equilibrium $\bar{y} \in(0,1)$, which is the only solution in $(0,1)$ of equation $(p x)^{n}+1=R_{0}(1-x)$.

(1) If condition (5.7) holds, then the positive equilibrium $\bar{y}$ is globally asymptotically stable.

(2) If condition (5.7) does not hold, then there exists $\tau^{*}>0$ such that $\bar{y}$ is asymptotically stable for $0<\tau<\tau^{*}$ and unstable for $\tau>\tau^{*}$, where $\tau^{*}$ is given by (4.3).

Corollary 5.9. If $0<p \leq 1$, then the positive equilibrium $\bar{y}$ of (5.6) is globally asymptotically stable for all $n>1$ and $R_{0}>1$.

Copyright $@$ by SIAM. Unauthorized reproduction of this article is prohibited. 
Proof. We have to show that

$$
\left(R_{0}-1\right) R_{0}^{\frac{n}{1-n}} \leq \frac{1}{n^{\frac{n}{n-1}}}+\frac{1}{n^{\frac{1}{n-1}}} .
$$

Define the map $w:(0, \infty) \rightarrow \mathbb{R}$ by $w(x)=(x-1) x^{n /(1-n)}, x>1$. It is easy to check that $w$ has a global maximum given by $w(n)=(n-1) n^{n /(1-n)}$. Hence,

$$
\left(R_{0}-1\right) R_{0}^{\frac{n}{1-n}} \leq(n-1) n^{n /(1-n)}=\frac{1}{n^{\frac{1}{n-1}}}-\frac{1}{n^{\frac{n}{n-1}}}<\frac{1}{n^{\frac{1}{n-1}}}+\frac{1}{n^{\frac{n}{n-1}}}
$$

which proves (5.11).

As in our second application, we can plot the stability diagrams for (5.6) showing the role of the different parameters. While the diagram $(p, \tau)$ is not substantially different from Figure 3, we find an interesting new feature for the stability charts involving the reproduction number $R_{0}$. In Figure 8 , we plot them for $n=2$. In contrast with Figure 4, for a given value of $p$ or $\tau$, we can ensure the global absolute stability of $\bar{y}$ for all values of $R_{0}>1$ sufficiently close to 1 . Thus, in some sense, we can affirm that the endemic bubble has a global character, which means that the equilibrium $\bar{y}$ loses its global stability, becomes unstable, and regains its global stability as $R_{0}$ is increased. We notice that for $n=2$ the endemic equilibrium $\bar{y}$ of (5.6) is globally stable for all values of $\tau$ and $R_{0}$ if $p<\sqrt{3}$, so an endemic bubble is only possible for $p>\sqrt{3}$. In Figure 9, we show a bubble for $n=2, p=2$, and $\tau=10$ in (5.6). We plotted it in the same way as for Figure 5.
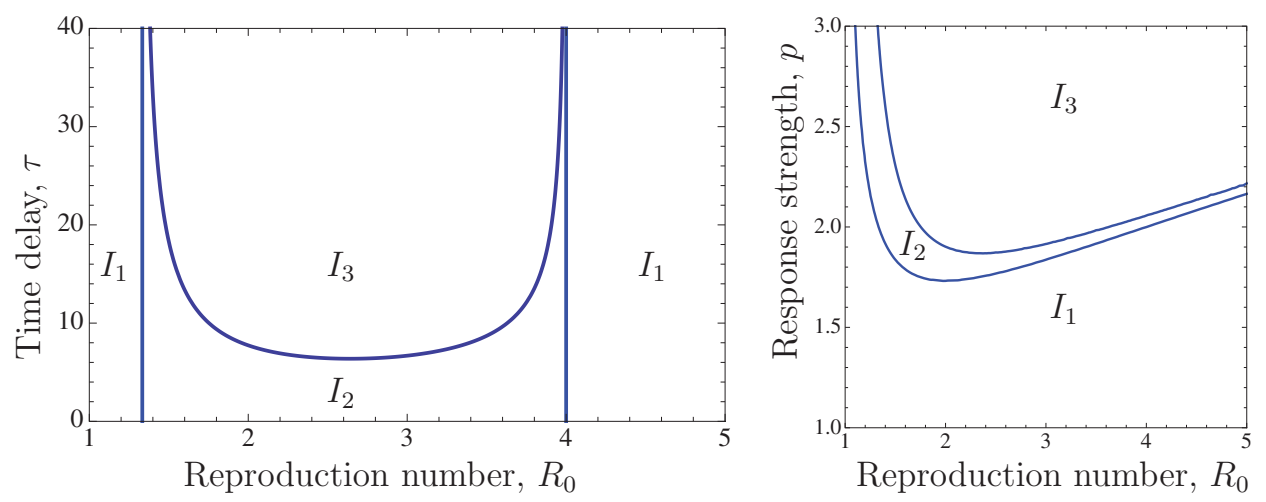

FIG. 8. Two-parameter stability diagrams for (5.6) with $n=2$. Left: for fixed $p=2$, dependence on the reproduction number $R_{0}$ and time delay $\tau$. Right: for fixed $\tau=10$, dependence on the reproduction number $R_{0}$ and the response strength $p$. In both cases, region $I_{1}$, determined by condition (5.7), corresponds to the absolute global stability of $\bar{y} ; \bar{y}$ is locally asymptotically stable in $I_{2}$; and $\bar{y}$ is unstable in $I_{3}$.

6. Conclusion. There have been many attempts in the disease modeling literature to incorporate the behavioral response of the members of a population to an epidemic into compartmental models, usually by modifying the transmission rate by a density-dependent factor. In this paper, we considered an SIS model with a very general reduction function $h$ (also called a media function in some studies), imposing only some natural hypotheses $(H)$, but assuming an explicit reaction delay in this response term. Then we obtain the delay differential equation (2.3), where the product 


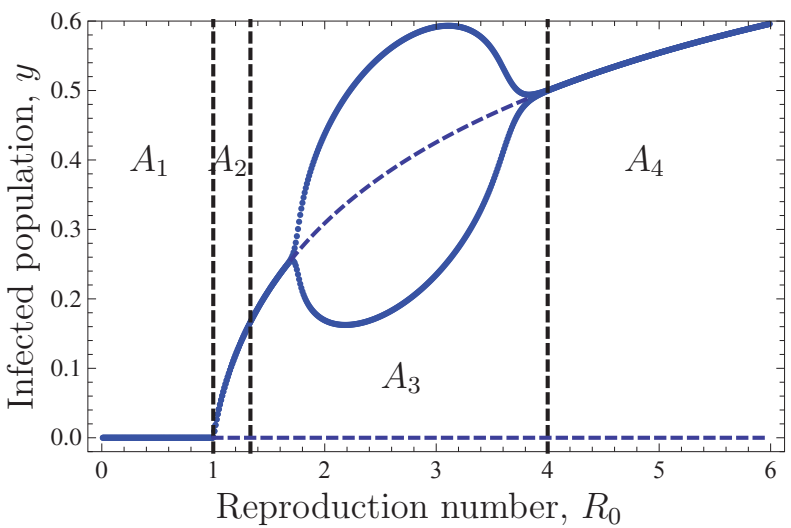

FIG. 9. Bifurcation diagram for (5.6) displaying an endemic bubble. In region $A_{1}\left(R_{0}<1\right)$, the disease-free equilibrium 0 is globally stable; in regions $A_{2}\left(1<R_{0}<4 / 3\right)$ and $A_{4}\left(R_{0}>4\right)$, the endemic equilibrium $\bar{y}$ is globally stable; in region $A_{3}\left(4 / 3<R_{0}<4\right), \bar{y}$ is asymptotically stable for $R_{0}<R_{*} \approx 1.753$, and $R_{0}>R^{*} \approx 3.629$, while for $R_{0} \in\left(R_{*}, R^{*}\right), \bar{y}$ becomes unstable and there are sustained oscillations (we plot the minimum and the maximum values of the limit cycle for each value of $R_{0}$ ). The equation undergoes two Hopf bifurcations at $R_{0}=R_{*}$ and $R_{0}=R^{*}$.

of delayed and nondelayed terms appears. We show that if the basic reproduction number $R_{0}$ is less than or equal to one, then the disease will be eradicated, while for $R_{0}>1$ it uniformly persists and a unique endemic equilibrium exists, regardless of the time delay $\tau$ or the specific form of the response function. More interestingly, we found various dynamics for $R_{0}>1$. Characterized by the properties of $h$ (see condition (4.2)), it is possible that the endemic equilibrium is stable for all values of $R_{0}$ and $\tau$, thus giving the usual simple picture as in Figure 1 (a) or Figure 2. In other cases, considering $R_{0}$ as a bifurcation parameter, we experience the loss of stability of the endemic equilibrium as $R_{0}$ increases, and a branch of periodic orbits emerges via a Hopf-bifurcation.

The most remarkable feature is that for large $R_{0}$, the endemic equilibrium always regains its stability and the periodic oscillation disappears, forming an interesting structure in the bifurcation diagram that we call (motivated by [9]) an endemic bubble; see Figures 5 and 9. To explore this phenomenon in detail, we considered the four most typical one-parameter families of reduction functions $(h(y)=1-p y$, $\left.h(y)=1-y /(p+y), h(y)=\exp (-p y), h(y)=1 /\left(1+(p y)^{n}\right)\right)$, each family being parametrized by a response strength $p$. We visualized the stability charts in terms of the relevant parameters $p, \tau$, and $R_{0}$, which reveals the nonmonotonic nature of the stability boundaries (see Figures 4 and 8), which leads to the bubbling effect. In addition, we have proved several global attractivity results for the endemic equilibrium. We note that, instead of the usual comparison techniques or Lyapunov functions, here we employed a technique for relating the delay differential equation to a discrete dynamical system, which is a novel application in the context of disease transmission models.

The estimates in Theorem 3.1 provide us an explicit lower and upper bound for the bubble, while by the stability results we can locate the bubble in the parameter space. More precise estimates and the finer structure of the bubble can be an interesting subject of further research (cf. [9]). The biological interpretation of the appearance of such a bubble is not immediately clear. We can give the following 
intuitive explanation: when $R_{0}$ is small, then the density of the infection remains at a low level, and thus the population does not react very strongly. The slope of $h$ represents how strong the reaction is to a small perturbation from the equilibrium state. For larger $R_{0}$, the endemic equilibrium is increased as well, and the population reduces the transmission rate, inducing oscillations by this delayed negative feedback. For very large $R_{0}$, however, the endemic equilibrium will be so large that individuals already reduced their transmission rates as much as they can, and further reduction is not feasible, resulting in a stable equilibrium. These properties are encoded into the shape of $h$.

A striking consequence of our results is the possibility of the following scenario: imagine that the system is beyond the bubble, for example, $R_{0}=4$ in the situation depicted in Figures 5 and 9. Then, after reducing the reproduction number by some other means, the system can move into the oscillatory regime with a high amplitude, so that the number of infected individuals can increase temporarily (and unexpectedly) to a higher level than it was before reducing $R_{0}$. On the other hand, it is possible that the minimum of the oscillatory solution goes near zero (such a situation can be seen in Figure 5 when $R_{0} \approx 2$ ), and in this scenario a relatively small intervention (for example, isolating all infected individuals, when there are not too many of them), if applied at the right time, has the potential to eradicate the disease even if $R_{0}$ is larger than one.

Acknowledgment. The authors are grateful to the anonymous referees for their helpful comments, which improved the presentation of this paper.

\section{REFERENCES}

[1] D. M. Bell and World Health Organization Working Group on Prevention of International and Community Transmission of SARS, Public health interventions and SARS spread, 2003, Emerg. Infect. Dis., 10 (2004), pp. 1900-1906.

[2] V. Capasso and G. Serio, A generalization of the Kermack-McKendrick deterministic epidemic model, Math. Biosci., 42 (1978), pp. 43-61.

[3] A. Cima, A. Gasull, And V. Mañosa, Dynamics of some rational discrete dynamical systems via invariants, Internat. J. Bifur. Chaos Appl. Sci. Engrg., 16 (2006), pp. 631-645.

[4] S. Collinson and J. Heffernan, Modelling the effects of media during an influenza epidemic, BMC Public Health, 14 (2014), 376.

[5] W. A. Coppel, The solution of equations by iteration, Proc. Cambridge Philos. Soc., 51 (1955), pp. 41-43.

[6] J. Cui, Y. Sun, And H. Zhu, The impact of media on the control of infectious diseases, J. Dynam. Differential Equations, 20 (2008), pp. 31-53.

[7] A. F. Ivanov, E. Liz, And S. Trofimchuk, Global stability of a class of scalar nonlinear delay differential equations, Differential Equations Dynam. Systems, 11 (2003), pp. 33-54.

[8] J. H. Jones And M. Salathe, Early assessment of anxiety and behavioral response to novel swine-origin influenza A(H1N1), PloS ONE, 4 (2009), e8032.

[9] T. Krisztin and E. Liz, Bubbles for a class of delay differential equations, Qual. Theory Dyn. Syst., 10 (2011), pp. 169-196.

[10] W. Liu, H. W. Hethcote, and S. A. Levin, Dynamical behavior of epidemiological models with nonlinear incidence rates, J. Math. Biol., 25 (1987), pp. 359-380.

[11] W. Liu, S. A. Levin, AND Y. IwaSA, Influence of nonlinear incidence rates upon the behavior of SIRS epidemiological models, J. Math. Biol., 23 (1986), pp. 187-204.

[12] M. Liu And G. Röst, Dynamics of an SIS model on homogeneous networks with delayed reduction of contact numbers, Biomath., 1 (2012), 1210045.

[13] M. LiU, G. Röst, AND G. VAS, SIS model on homogeneous networks with threshold type delayed contact reduction, Comput. Math. Appl., 66 (2013), pp. 1534-1546.

[14] R. Liu, J. Wu, AND H. ZHu, Media/psychological impact on multiple outbreaks of emerging infectious diseases, Comput. Math. Methods Med., 8 (2007), pp. 153-164.

[15] E. LIz, Local stability implies global stability in some one-dimensional discrete single-species 
models, Discrete Contin. Dyn. Syst. Ser. B, 7 (2007), pp. 191-199.

[16] E. Liz AND G. Röst, Dichotomy results for delay differential equations with negative Schwarzian derivative, Nonlinear Anal. Real World Appl., 11 (2010), pp. 1422-1430.

[17] E. Liz And G. Röst, Global dynamics in a commodity market model, J. Math. Anal. Appl., 398 (2013), pp. 707-714.

[18] M. E. J. Newman, The structure and function of complex networks, SIAM Rev., 45 (2003), pp. $167-256$.

[19] R. Pastor-Satorras and A. Vespignani, Epidemic dynamics and endemic states in complex networks, Phys. Rev. E, 63 (2001), 066117.

[20] G. Röst AND J. Wu, Domain-decomposition method for the global dynamics of delay differential equations with unimodal feedback, Proc. R. Soc. Lond. Ser. A Math. Phys. Eng. Sci., 463 (2007), pp. 2655-2669.

[21] D. Singer, Stable orbits and bifurcation of maps of the interval, SIAM J. Appl. Math., 35 (1978), pp. 260-267.

[22] H. Smith, An Introduction to Delay Differential Equations with Sciences Applications to the Life, Springer, 2010.

[23] C. Sun, W. Yang, J. Arino, and K. Khan, Effect of media-induced social distancing on disease transmission in a two patch setting, Math. Biosci., 230 (2011), pp. 87-95.

[24] Q. Wu, X. Fu, M. Small, And X. J. XU, The impact of awareness on epidemic spreading in networks, Chaos, 22 (2012), 013101.

[25] Y. XiaO, X. XU, AND S. TANG, Sliding mode control of outbreaks of emerging infectious diseases, Bull. Math. Biol., 74 (2012), pp. 2403-2422.

[26] Y. Xiao, T. Zhao, And S. TANG, Dynamics of an infectious diseases with media/psychology induced non-smooth incidence, Math. Biosci. Eng., 10 (2013), pp. 445-461.

[27] J. Yorke And W. P. London, Recurrent outbreaks of measles, chickenpox and mumps II. Systematic differences in contact rates and stochastic effects, Amer. J. Epidemiology., 98 (1973), pp. 469-482.

[28] H. ZhaO, Y. Lin, AND Y. DAI, An SIRS epidemic model incorporating media coverage with time delay, Comput. Math. Methods Med., 2014 (2014), 680743.

Copyright $@$ by SIAM. Unauthorized reproduction of this article is prohibited. 